\title{
Deskewing by space-variant deblurring
}

Karthik Seemakurthy

karthikvjit@gmail.com

Subeesh Vasu

ee13d050@ee.iitm.ac.in

\author{
A.N. Rajagopalan \\ raju@ee.iitm.ac.in
}

Image Processing and Computer Vision

Lab

Department of Electrical Engineering

IIT Madras

Chennai, India
Skew and motion blur are significant challenges when camera and scene of interest are in two different media. Skew occurs due to spatially varying refraction on a dynamic water surface, whereas motion blur results from multiple intensities impinging on the imaging sensor during camera exposure time due to time varying refraction. In this paper, we propose a technique to restore underwater images degraded by attenuated water waves. Following others [4] [2], we also assume that the static camera is looking vertically downwards and imaging a planar scene but through spatially decaying periodic water waves (such as waves due to breeze in shallow water bodies). Also, we allow for the attenuation factor as well as the direction of the water waves to undergo changes during the exposure time of the camera (a situation not handled by the state-ofthe-art work in [3]). We propose a shot detection method that automatically segments a captured video into groups of frames wherein each group is governed by a dominant water wave direction (i.e. unidirectional) and a single exponential factor of attenuation. Within each segment, we average the frames and show that the blur induced at different pixel locations are scaled versions of each other and model these scale factors through a virtual depth map. Note that despite the scene being planar, the blur induced due to attenuated water waves is space-variant in nature. We pose deskewing of the planar scene as equivalent to a space-variant deblurring problem corresponding to a $3 \mathrm{D}$ scene with depth profile being the same as that of the virtual depth map. We propose an alternating framework to solve for the latent image from a single blurred observation. The procedure can likewise be repeated for other segments too, if needed.

We compare our latent image estimation results with the state-of-the-art space-variant deblurring approach [5] along with state-of-the-art deskewing methods [4], [2]. The input to [4] [2] should be in the form of video, while a single blurred image is used for [5] and our proposed algorithm. For synthetic experiments, we use the model described in [1] to simulate a decaying envelope of the water waves which is given as $h(\mathbf{x}, t)=A(\mathbf{x}) \sin \left(\omega_{x} \mathbf{x}+\omega_{y} \mathbf{y}-t\right)$, where $\mathrm{A}(\mathbf{x})$ denotes the decaying amplitude of the sinusoid, $\omega_{x}$ and $\omega_{y}$ are the spatial frequencies. In all our experiments, the decaying function is given by $A(\mathbf{x})=A_{0} \exp \left(d_{f} \cdot\left(\mathbf{d}_{\mathbf{d}}{ }^{T} \mathbf{x}\right)\right)$, where $d_{f}$ is the decay factor and $\mathbf{d}_{\mathbf{d}}$ is the decay direction. We used laminated textured sheets kept at the bottom of an aquarium for indoor experiments. The field-of-view was kept large enough to witness the effect of wave attenuation in the captured video. The source of waves is a fan that acts as a wind blower. We additionally performed outdoor experiments in swimming pool. Here the source of waves was the breeze as well the flow due to water circulation. For all examples, our method outperformed competitive methods, qualitatively and quantitatively.

[1] An-Kuo Liu and Stephen H Davis. Viscous attenuation of mean drift in water waves. Journal of Fluid Mechanics, 81(01):63-84, 1977.

[2] Omar Oreifej, Guang Shu, Teresa Pace, and Mubarak Shah. A two-stage reconstruction approach for seeing through water. In Computer Vision and Pattern Recognition (CVPR), 2011 IEEE Conference on, pages 1153-1160. IEEE, 2011.

[3] Karthik Seemakurthy and Ambasamudram Narayanan Rajagopalan. Deskewing of underwater images. Image Processing, IEEE Transactions on, 24(3):1046-1059, 2015.

[4] Yuandong Tian and Srinivasa G Narasimhan. Seeing through water: Image restoration using modelbased tracking. In Computer Vision, 2009 IEEE 12th International Conference on, pages 23032310. IEEE, 2009.

[5] Li Xu, Shicheng Zheng, and Jiaya Jia. Unnatural 10 sparse representation for natural image deblurring. In Proceedings of the IEEE Conference on Computer Vision and Pattern Recognition, pages 1107-1114, 2013. 